\title{
Improving the radiation hardness of space solar cells via nanophotonic light trapping
}

\author{
A. Mellor ${ }^{1}$, N. P. Hylton ${ }^{1}$, Ch. Wellens ${ }^{2}$, T. Thomas ${ }^{1}$, Y. Al-Saleh ${ }^{1}$, V. Giannini ${ }^{1}$, A. Braun ${ }^{1}$, H. Hauser ${ }^{2}$, \\ S. A. Maier ${ }^{1}$, N. J. Ekins-Daukes ${ }^{1}$ \\ ${ }^{1}$ Department of Physics, Imperial College London, London SW7 2AZ, United Kingdom \\ ${ }^{2}$ Fraunhofer Institute for Solar Energy Systems ISE, Heidenhofstr. 2, 79110 Freiburg, Germany
}

\begin{abstract}
We show that the radiation-hardness of space solar cells can be significantly improved by employing nanophotonic light trapping. Two light-trapping structures are investigated in this work. In the first, an array of Al nanoparticles is embedded within the anti-reflection coating of a GaInP/InGaAs/Ge solar cell. A combined experimental and simulation study shows that this structure is unlikely to lead to an improvement in radiation hardness. In the second, a diffractive structure is positioned between the middle cell and the bottom cell. Computational results, obtained using an experimentally validated electro-optical simulation tool, show that a properly designed light-trapping structure in this position can lead to a relative $10 \%$ improvement in the middle-cell photocurrent at end-of-life.
\end{abstract}

\section{INTRODUCTION}

In space, solar cells are subject to high-energy electron and proton radiation, which damages the semiconductor material leading to degradation in efficiency[1]. The most notable degradation is a reduction photocurrent due to reduction of the minority carrier diffusion length. In the $\mathrm{GaInP} / \mathrm{InGaAs} / \mathrm{Ge}$ solar cells presently favoured for use in space, this effect is most pronounced in the InGaAs middle cell, whose photocurrent limits the overall cell efficiency at end of life (EOL) [2].

In this work, we show that the radiation-hardness of the InGaAs middle cell can be significantly improved by employing nanophotonic light trapping. Light trapping allows the subcell thickness to be significantly reduced, whilst maintaining strong photon absorption throughout the middlecell absorption band. This reduces the average distance each carrier needs to diffuse to reach the junction, thus improving the photocurrent at EOL.

Two light trapping structures are investigated in this work. The first (Structure 1) consists of an array of Aluminium nanoparticles embedded in the anti-reflection coating (ARC) of a monolithic solar cell. Through an experimental and simulation study, it is shown that this type of front-side structure is unlikely to improve the EOL performance of space solar cells. The second (Structure 2) is a diffractive structure positioned between the middle and bottom subcells in a bonded four-terminal device. Here, simulation results show that a properly designed light-trapping structure can lead to a relative $15 \%$ improvement in the middle-cell photocurrent at EOL.

\section{METHODS}

\section{A. Experimental}

Triple-junction space solar cells with full metallization but no ARC were provided by AzurSpace. Double-layer $\mathrm{MgF}_{2} / \mathrm{TaO}_{\mathrm{x}}$ ARCs with $\mathrm{Al}$ nanoparticle arrays embedded within the $\mathrm{TaO}_{\mathrm{x}}$ layer were then fabricated. The nanoparticle arrays were realized over the whole solar cell area by nanoimprint lithography [3]. The nanoparticles had an array period of 300 $\mathrm{nm}$, a diameter of $150 \mathrm{~nm}$ and a height of $15 \mathrm{~nm}$. The nanoparticles were separated from the semiconductor by a 15 nm thick $\mathrm{TaO}_{\mathrm{x}}$ spacer layer. A control sample with identical ARC layers but no nanoparticles was also produced.

The cells were irradiated with a $1 \times 10^{15} \mathrm{~cm}^{-2}$ fluence of $1 \mathrm{MeV}$ electrons, followed by photon annealing under AM0 at $25 \mathrm{C}$ for 60 hours, then dark annealing at $60^{\circ} \mathrm{C}$ for 24 hours. The EQE of the devices was measured before and after irradiation testing; these results are denoted beginning of life (BOL) and end of life (EOL) respectively.

\section{B. Computational}

An electro-optical simulation tool has been developed to model multi-junction solar cells employing a periodic light light-trapping nanostructure located somewhere in the device. The method is based on coupling an optical rigorous coupled wave analysis (RCWA) model to a one-dimensional electrical drift-diffusion model. The former is implemented using commercial package GdCalc®[4] and the latter is semianalytical[5]. This combined model is a simplification of that in Refs. [6, 7]. The calculation yields the expected external quantum efficiency (EQE) of the simulated device. The effect of irradiation is accounted for in the electrical part of the model via the minority carrier diffusion lengths in the InGaP and InGaAs subcells. The simulation tool is validated against experimental data in section III.

\section{RESULTS}

\section{A. Structure 1 - Nanoparticles embedded in ARC}

The solid curves in Figure 1 show the measured top- and middle-cell EQEs for Sample Set 2. Since these samples were irradiated, results are shown both at BOL (a) and EOL (b). 
Here, the nanoparticles lead to a broadband loss of 10 and $20 \%$ absolute in the top- and middle-cells respectively. This is observed both at BOL and EOL. We conclude that the nanoparticle array has not improved the EOL photocurrent of either subcell. Figure 1 also shows good agreement between experiment and simulation; we consider this a validation of the simulation tool.
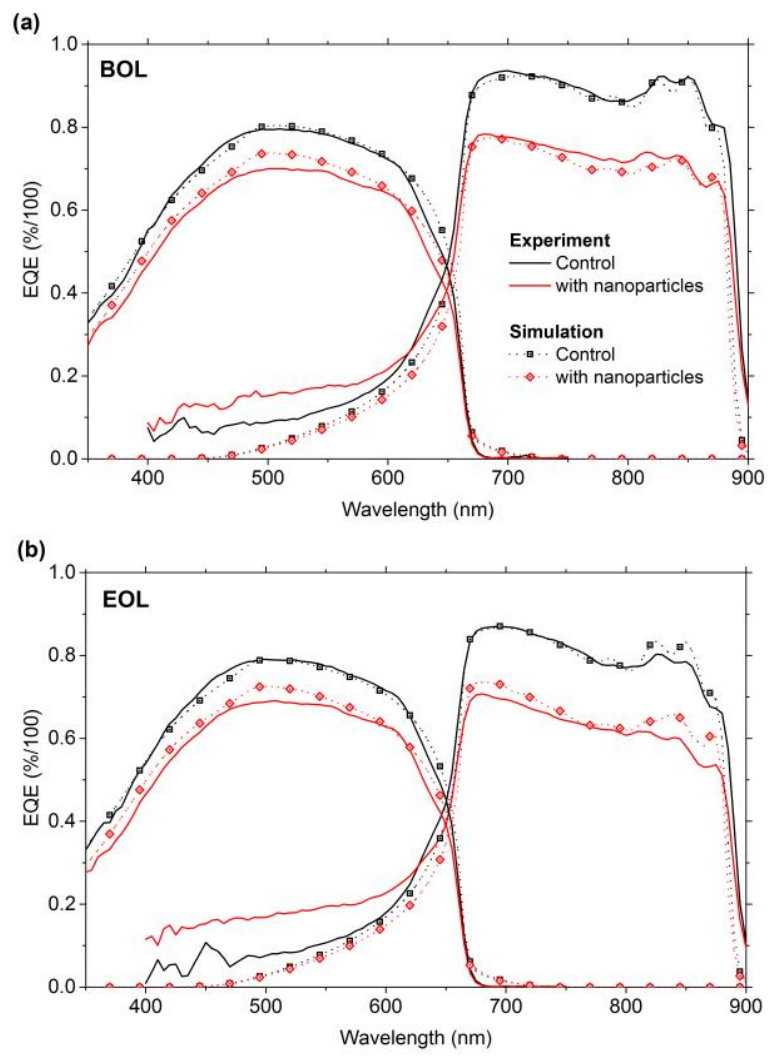

Figure 1. Measured and Simulated EQE for Sample Set 2. The nanoparticles have a diameter of $150 \mathrm{~nm}$, a height of $15 \mathrm{~nm}$ and an array period of $300 \mathrm{~nm}$. (a): before irradiation exposure (BOL). (b): after irradiation exposure (EOL).

To form more general conclusions regarding front-side nanoparticle arrays for multi-junction solar cells, the electrooptical simulation has been used to study a range of nanoparticle-array dimensions and positions in the ARC. Simulations were made for the three configurations, shown in Figure 2; these being the nanoparticles embedded in the ARC bottom layer (Configuration 1), embedded in the ARC top layer (Configuration 2) and sitting on top of the ARC (Configuration $3)$. For each configuration, simulations have been made over a range of array periods, $\Lambda$, nanoparticle diameters, $D$, and nanoparticle heights, $H$. Nearly 6000 spectra were calculated, taking in every permutation of the three-dimensional parameter space encompassing the ranges $200<\Lambda<400 \mathrm{~nm}, 20<r<190$ $\mathrm{nm}$ and $10<h<50 \mathrm{~nm}$, with a $10 \mathrm{~nm}$ step size in each case. A planar benchmark structure was also simulated, which has an identical ARC but no nanoparticles. In all cases the ARC is $\mathrm{AlO}_{\mathrm{x}} / \mathrm{TiO}_{\mathrm{x}}$.

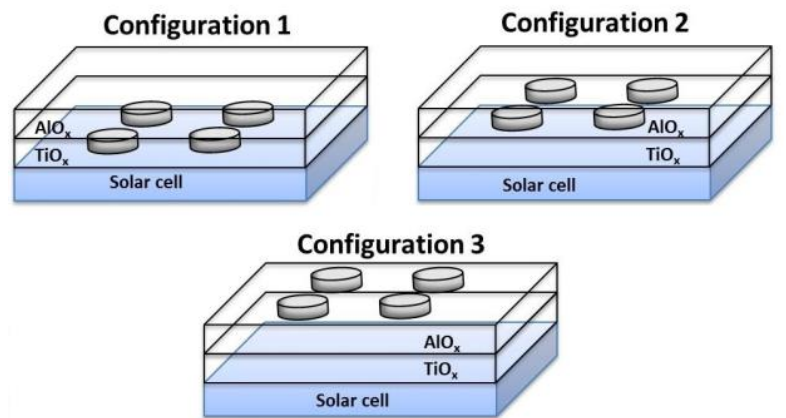

Figure 2. Schematic of the three configurations for embedding the nanoparticles in the ARC.

Figure 3 shows a scatterplot with the calculated photocurrent in the $\mathrm{In}_{0.01} \mathrm{GaAs}$ middle cell as the y-ordinate, and the total volume of nanoparticles as a fraction of the ARC volume (proportional to $h \pi \mathrm{r}^{2} \Lambda^{-2}$ ) as the $\mathrm{x}$-ordinate. Note that many combinations of array dimensions can have the same volume fraction, and that the currents shown are much lower than for state-of-the-art-cells (even for the benchmark), due to the thin $\mathrm{In}_{0.01} \mathrm{GaAs}$ subcell.

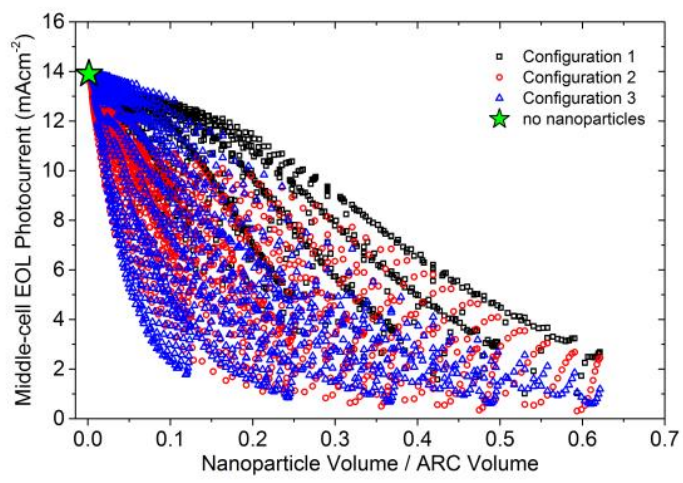

Figure 3. Scatter plot showing the $\operatorname{In}_{0.01}$ GaAs middle cell photocurrent $\mathrm{Vs}$ the fractional volume of nanoparticles

It can be seen that, for all configurations, nanoparticle-array dimensions and materials, the calculated photocurrent is never greater than for the planar benchmark (shown as a green star). Introducing metallic nanoparticles anywhere in the ARC generally leads to a decrease in photocurrent if the particles occupy any appreciable volume.

To better understand these results, the average spectrumweighted IQE and transmission into the solar cell have been calculated: denoted $I Q E_{\Phi}$ and $T_{\Phi}$ respectively. For each simulation result, $I Q E_{\Phi}$ is plotted against $T_{\Phi}$ in the scatterplot in Figure 4 . This shows clearly the trade-off between transmission into the solar cell and oblique scattering. Also shown in Figure 4 is a break-even line. This is a locus of points for which the product $T_{\Phi} \cdot I Q E_{\Phi}$ is equal to the same product for the planar benchmark. Any simulation result above this line would yield a net improvement over the planar benchmark. We can see that, for many nanoparticle-array dimensions, the IQE is improved. We attribute this to the light-trapping effect. However, this comes at an even greater loss in transmission into the solar cell, 
yielding an overall loss. This study is expanded on in a pending journal paper[8].

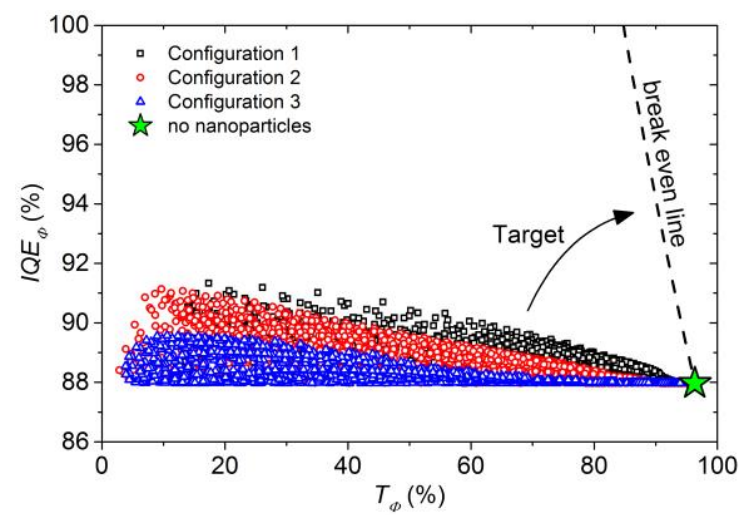

Figure 4. Scatter plot showing $I Q E_{\Phi}$ Vs. $T_{\Phi}$ for every combination of dimensions in each configuration.

\section{B. Structure 2 - diffractive structure positioned between the} middle and bottom subcells

Here, the validated electro-optical simulation tool is used to investigate how a diffractive structure positioned between the middle and bottom subcells (Structure 2) can improve the radiation hardness of the InGaAs middle cell. The positioning of the diffractive structure is shown in Figure 5 (a). This is compared to a benchmark solar cell shown in Figure 5 (b). The benchmark incorporates a Bragg mirror between the middle and bottom subcells. The InGaAs middle-cell thickness is 1750 for the benchmark solar cell and $700 \mathrm{~nm}$ for the solar cell incorporating Structure 2. Exact details of structure 2 can be found in a pending journal paper[9].

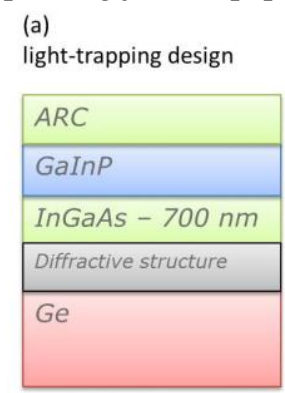

(b)

benchmark

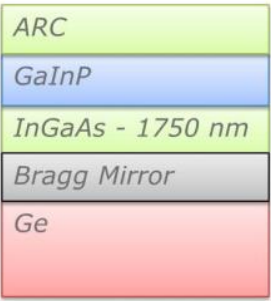

Figure 5. Schematic of the positioning of structure 2 (a), and of the benchmark solar cell to which it is compared (b).

Figure 6 shows the calculated absorption in the InGaAs middle cell for the benchmark and structure 2. It can be seen that both cells have a similar overall absorption, shown that the presence of structure 2 allows thinning of the middle cell whilst maintaining high absorption.

Figure 7 shows the middle cell photocurrent as a function of $1 \mathrm{MeV}$ electron fluence. It can be seen that the presence of Structure 2 significantly improves the radiation hardness of the middle cell. After $1 \times 10^{15} \mathrm{~cm}^{-2}$ fluence, a $15 \%$ relative improvement in the middle cell photocurrent is predicted compared to the benchmark.

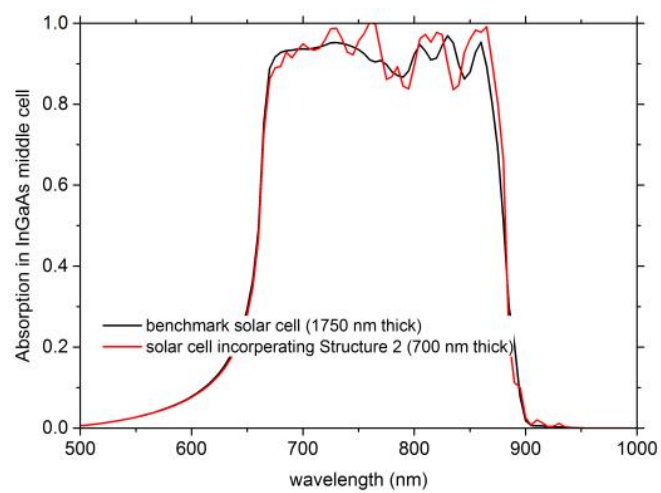

Figure 6. Calculated absorption in the InGaAs middle cell for Structure 2 and the benchmark.

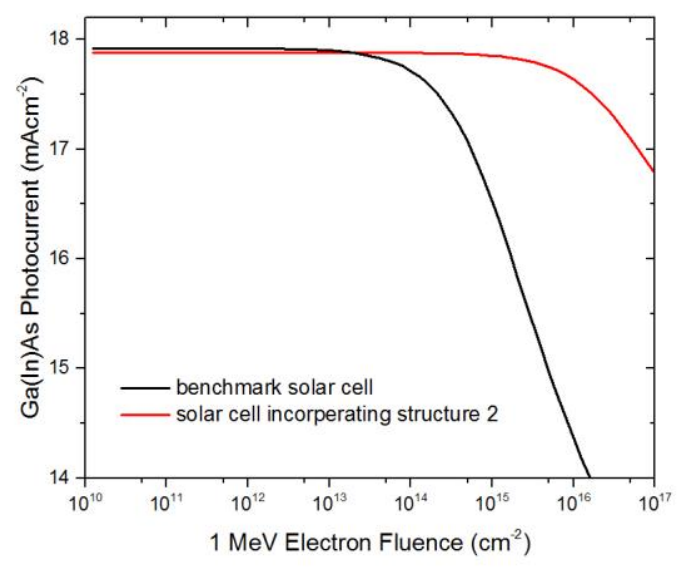

Figure 7. Predicted InGaAs middle cell photocurrent as a function of $1 \mathrm{MeV}$ electron fluence.

\section{REFERENCES}

[1] M. Yamaguchi, Radiation resistance of compound semiconductor solar cells, Journal of Applied Physics, 78 (1995) 1476-1480.

[2] T. Takamoto, T. Agui, K. Kamimura, M. Kaneiwa, Multijunction solar cell technologies - high efficiency, radiation resistance, and concentrator applications, in: Photovoltaic Energy Conversion, 2003. Proceedings of 3rd World Conference on, 2003, pp. 581-586 Vol.581.

[3] H. Hauser, N. Tucher, K. Tokai, P. Schneider, C. Wellens, A.K. Volk, S. Seitz, J. Benick, S. Barke, F. Dimroth, C. Müller, T. Glinsner, B. Bläsi, Development of nanoimprint processes for photovoltaic applications, Journal of Micro/Nanolithography, MEMS, and MOEMS, 14.3 (2015) 031210-031210.

[4] K. Johnson, Grating Diffraction Calculator (GD-Calc $®)$, in, kjinnovation, Santa Clara, CA 95051, 2005.

[5] J. Nelson, The Physics of Solar Cells, Imperial College Press, 2003.

[6] X. Li, N.P. Hylton, V. Giannini, K.-H. Lee, N.J. EkinsDaukes, S.A. Maier, Multi-dimensional modeling of solar cells 
with electromagnetic and carrier transport calculations, Progress in Photovoltaics: Research and Applications, 21 (2013) 109-120.

[7] X. Li, N.P. Hylton, V. Giannini, K.-H. Lee, N.J. EkinsDaukes, S.A. Maier, Bridging electromagnetic and carrier transport calculations for three-dimensional modelling of plasmonic solar cells, Opt. Express, 19 (2011) A888-A896.

[8] A. Mellor, N.P. Hylton, H. Hauser, T. Thomas, K.H. Lee, Y. Al-Saleh, V. Giannini, A. Braun, J. Loo, D. Vercruysse, P. Van Dorpe, B. Bläsi, S.A. Maier, N.J. Ekins-Daukes, Nanoparticle scattering for multi-junction solar cells: the tradeoff between absorption enhancement and transmission loss, under review, IEEE Journal of Photovoltaics, (2016).

[9] A. Mellor, N.P. Hylton, S.A. Maier, N. Ekins-Daukes, Interstitial light-trapping design for multi-junction solar cells, Progress in Photovoltaics: Research and Applications, under review (2016). 